\title{
Asistencia personal en la autoeficacia, calidad de vida y estilo de afrontamiento en personas con diversidad funcional de origen físico
}

\author{
Coral García Hortal ${ }^{1}$, Katja Villatoro Bongiorno², Marta Sahagún Navarro³ \\ Recibido: 27-02-2015 - Aceptado: 19-05-2015
}

\begin{abstract}
Resumen
El objetivo de este estudio fue analizar la autoeficacia, la calidad de vida y los estilos de afrontamiento en personas con diversidad funcional de origen físico, para comprobar la influencia de estas con el apoyo de asistencia personal (prestación económica de la Ley 39/2006 de España) y sin él, en una muestra total de 65 personas. La hipótesis que se planteó fue que las personas con diversidad funcional de origen físico que tienen la prestación de asistencia personal tienen mayor calidad de vida, más autoeficacia y mejor estilo de afrontamiento ante las diferentes situaciones cotidianas. Para ello, se creó un cuestionario compuesto por tres instrumentos diferentes, uno por cada una de las variables escogidas: la Escala de Autoeficacia General (Baessler y Schwarzer, 1996), el cuestionario Whoqol-Bref (Organización Mundial de la Salud, 1996) y el cuestionario Brief-COPE (Carver, 1997). Tras la recogida de datos, se realizó un análisis univariado para la descripción de la muestra y de las variables principales del estudio, y un análisis bivariado para establecer las posibles diferencias entre los grupos de análisis. Los resultados muestran que los niveles de autoeficacia y algunos de los dominios de calidad de vida son más altos en las personas que cuentan con asistencia personal que en las que no, pero no se observan diferencias en los estilos de afrontamiento.
\end{abstract}

Palabras clave: autoeficacia, estilos de afrontamiento, calidad de vida, asistencia personal, diversidad funcional de origen físico.

\section{Personal assistance in self-efficacy, quality of life and coping style in people with functional diversity of physical}

\begin{abstract}
The aim of this study was to analyze the efficacy, quality of life and styles coping in people with functional diversity of physical origin, checking the influence of these with the support of personal assistance (economic benefit of Law 39/2006 of Spain) and without it, in a total sample of 65 people. The hypothesis raised was that people with functional diversity of physical origin that have the provision of personal

${ }^{1}$ Graduada en Psicología por la Universidad de Sevilla, España; coralgarciahortal@hotmail.com

${ }^{2}$ Doctoranda y Magíster en Atención socio-sanitaria a la dependencia por la Universidad de Valencia, España. Diplomada en Salud Pública. Enfermera. Docente en Cruz Roja, España; katvibon@gmail.com

${ }^{3}$ Doctoranda y Magíster en Atención socio-sanitaria a la dependencia por la Universidad de Valencia, España. Trabajadora social. Docente-Investigadora en Corporación Universitaria del Caribe -CECAR, Colombia; marta.sahagun@cecar.edu.co
\end{abstract}


BÚSQUEDA - Enero / Junio de 2015 - No. 14 (7 - 18)

care has improved quality of life, more efficacy and better way of coping with everyday situations. To do this, a questionnaire is created composed of three different instruments, one for each of the variables: selected General Self-Efficacy Scale (Baessler y Schwarzer, 1996), the WHOQOL-BREF (World Health Organization, 1996) and Brief-COPE questionnaire (Carver, 1997). After data collection, was performed a univariate analysis for the description of the sample and the main variables of the study, and bivariate to establish possible differences between groups analysis. The results show that levels of self-efficacy and some of the domains of quality of life are higher in people who have personal assistance at no but no differences in coping styles are observed.

Keywords: self-efficacy, coping styles, quality of life, personal care, functional diversity of physical origin.

\section{Introducción}

Las personas con discapacidad física o diversidad funcional han sido discriminadas a lo largo de la historia. Los tres modelos que se han seguido en el trato de la discapacidad han sido: el modelo de prescindencia, que otorga una causa religiosa al origen la discapacidad y considera a estas personas como prescindibles; el modelo rehabilitador, que toma lo científico como causa y busca la rehabilitación de las personas con discapacidad; y el modelo social, que busca las causas en la sociedad y aspira a la igualdad pero sin olvidar que estas personas siguen siendo diferentes.

El modelo social defiende que la concepción de la discapacidad es una construcción social impuesta, y plantea una visión de la discapacidad como clase oprimida, con una severa crítica al rol desempeñado por los profesionales y la defensa de una alternativa de carácter político más que científico (Verdugo, s.f.).

Del mismo modo, existen referencias al cambio del término de discapacidad, ya que se su evolución futura, parece centrarse en el empleo de la expresión personas con necesidad de apoyo, aunque esto todavía no aparece reflejado en la legislación española (Verdugo, Vicent, Campo y Jordan de Urríes, 2001).

En el campo de la psicología, al igual que en el resto de campos científicos, esta población empezó a ser estudiada desde la perspectiva médica o rehabilitadora; considerando que las limitaciones de las personas con discapacidad vienen determinadas por una enfermedad y que ellas deben ser curadas o rehabilitadas.

Desde la psicología el estudio se centraba en el trastorno o en la enfermedad, que causaban a su vez limitaciones en la vida diaria. Así, por ejemplo, una persona que tuviera una lesión ocular no podría ver y, por tanto, tendría una serie de limitaciones que requerirían nuevos aprendizajes. De hecho, solo hasta mediados del siglo XX es cuando la discapacidad empieza a ser afrontada desde las disciplinas sociales.

En España, el artículo 14 de la Constitución de 1978 establecía que todos somos iguales ante la ley. A partir de este momento, se crearon normas y leyes destinadas a que las personas con discapacidad pudieran alcanzar la integración y la normalización en la sociedad.

Sin embargo, aunque en las últimas décadas se ha avanzado en la lucha por la igualdad de toda la población, las personas con discapacidad están todavía muy lejos de alcanzar la plena inclusión en la sociedad. De esta forma, la mayoría de quienes poseen discapacidad sigue sin poder realizar tareas tan cotidianas para los demás como ir al cine, entrar en un bar o usar cualquier servicio público porque estos no son accesibles para ellos.

Tampoco están plenamente incluidos en las escuelas o las universidades; muchos no realizan estudios superiores o no trabajan porque constantemente se les interponen barreras que se lo impiden. Incluso a día de hoy, su realidad es plenamente discriminatoria y los modelos 
actuales de análisis de esta realidad se han mostrado insuficientes a la hora de erradicar esa discriminación y a la hora de afrontar los nuevos retos bioéticos (Palacios y Romañach, 2007).

En definitiva, la discriminación de los discapacitados, intencionada o no, se produce en todos los ámbitos de la vida diaria, aunque la mayoría de las veces estas dificultades solo son visibles para quienes son discapacitados o para las personas cercanas a ellos. Y esto sucede en un país en el que el diez por ciento de la población tiene alguna discapacidad.

Todo esto impulsa el surgimiento, desde el modelo social, de una nueva perspectiva en el contexto de las personas con discapacidad, física generalmente, y vinculadas al Movimiento de Vida Independiente.

Desde el modelo social, se considera que la discapacidad no es inherente a la persona, sino causada por las delimitaciones del entorno; y desde el movimiento de vida independiente, se entiende que todas las personas son diversas en las funciones, centrándose así en la variedad y no en la diferencia o límites.

Además, en el Movimiento de Vida Independiente, se interpreta que todos los seres humanos funcionan de forma diferente que, con los apoyos adecuados y suficientes nunca supondría ninguna consecuencia negativa para nadie; en cambio, si no se recibe el apoyo citado, la sociedad les convertirá en discapacitados y no podrán ser partícipes de ella.

Esta perspectiva está siendo adoptada ya en algunos de los ámbitos de la psicología y de otras ciencias. De esta forma, el estudio no se centraría en el trastorno o enfermedad, que son la causa de las dificultades y generan una sociedad excluyente, lo que causa a su vez unas limitaciones por falta de oportunidades. De tal manera que, por ejemplo, una persona con daños oculares tendría unas limitaciones si no se le proporcionan los instrumentos adecuados: unas gafas.
Es por todo ello, por lo que este movimiento aboga por el uso de un nuevo término para referirse al colectivo de las personas con discapacidad: diversidad funcional, frente a los peyorativos y obsoletos personas con discapacidad, discapacitados, minusvalía, minusválidos, deficiencia o invalidez.

Este trabajo se centra en el modelo surgido desde el Movimiento de Vida Independiente, pues es, actualmente, la perspectiva más innovadora desde la psicología social, la cual tiene un papel fundamental, pues se evidencia como necesaria la actuación social con el objetivo de adaptar la sociedad para que sea también incluyente las personas con diversidad funcional.

Desde las clasificaciones internacionales de la salud también se observa un puente tendente hacia el cambio de un nuevo enfoque. En este sentido, se define como un puente entre el modelo médico-rehabilitador y el modelo social, cambiando así la denominación que durante años ha esgrimido.

La clasificación que se hace en el 2001, apoyada en los nuevos enfoques, ya no se denomina Clasificación Internacional de la Deficiencia, la Discapacidad y Minusvalía (CIDDM), sino Clasificación Internacional del Funcionamiento, la Discapacidad y la Salud conocida como CIF [...] La CIF proporciona un perfil útil del funcionamiento de la persona en situación de discapacidad y de la salud del individuo en varios dominios. La CIF se divide en dos: Clasificación Internacional de la Enfermedad (CIE) y Clasificación Internacional del Funcionamiento (CIF), la cual dio pie a considerar que la mirada no solo debe ser desde la salud, sino que hay muchas otras disciplinas que deben reflexionar sobre el funcionamiento de dicha población. (Soto, 2008, p. 14).

Desde el campo de la psicología social, se pretende centrar este trabajo en estudiar qué apoyos son los elegidos por las personas con diversidad funcional para formar parte de la sociedad de una manera normalizada. Históricamente, el único apoyo social que han recibido las personas con discapacidad son los centros residenciales, lugares donde deben permanecer muchas veces durante años debido a la elección de sus familias y donde no cuentan 
BÚSQUEDA - Enero / Junio de 2015 - No. 14 (7 - 18)

con capacidad de autodeterminación. También ha sido frecuente el uso de la ayuda a domicilio ó los cuidados familiares.

Sin embargo, existe un derecho legislado a nivel del Estado español a través del texto refundido de la Ley General de Discapacidad (2013), e internacionalmente con la Convención de los Derechos Humanos de las personas con discapacidad, de la Organización de las Naciones Unidas (World Health Organization, 2006), que les facilita vivir con independencia a la vez que favorece su inclusión en la sociedad y su capacidad de autodeterminación: la asistencia personal.

De este modo, la asistencia personal, prestación legislada por la Ley 39/2006 de promoción de la autonomía personal y atención a las personas en situación de dependencia, está reconocida como un derecho fundamental, en la Convención de los Derechos Humanos de las personas con discapacidad que España firmó y ratificó, siendo esta de rango internacional y de obligado cumplimiento, comprometiéndose a cumplirla en 2008.

Esta prestación consiste en proporcionar un asistente a las personas con discapacidad para que las apoye en todas aquellas áreas en las que necesiten ayuda, de forma que puedan llevar una vida independiente, en sus propias casas, barrios, con sus círculos de amigos y realizando aquellas actividades que hayan elegido por voluntad propia (ir a la Universidad, viajar, pasear...).

Según el Movimiento de Vida Independiente es el propio usuario quien escoge su asistente personal y gestiona el servicio, es decir, gestiona y controla su propia vida. En algunas de las Comunidades Autónomas de nuestro país se ha desarrollado esta ley más que en otras, donde no se ha desarrollado lo suficiente o nada. Por ejemplo, en Andalucía solo cuentan con este apoyo quince personas y, sin embargo, en el País Vasco este número asciende a más de mil (IMSERSO, 2015).

Después de haber introducido el tema de asistencia personal para personas con diversidad funcional física como medida de apoyo para poder obtener calidad de vida e independencia, es necesario hacer énfasis a las distintas variables psicológicas objeto de este estudio: autoeficacia, calidad de vida y estilos de afrontamiento, que se describen a continuación.

La autoeficacia se introdujo por primera vez como concepto por Bandura en 1977 y lo definió como "las creencias en la propia capacidad para organizar y ejecutar las acciones requeridas para manejar las situaciones futuras" (Bandura, 1999, p. 21). Posteriormente, en su libro Fundamentos sociales del pensamiento y la acción (1986), propone la teoría social-cognitiva que enfatiza el papel de los fenómenos autorreferenciales (lo que uno se dice a sí mismo) como el medio por el cual el hombre es capaz de actuar en su ambiente.

Además, una de las tareas más importantes para la psicología es comprender la manera en que los seres humanos se adaptan y logran sus retos cotidianos (Cantó, 1998). De esta manera, la autoeficacia es la percepción de la capacidad de uno mismo para realizar una tarea, por lo que puede convertirse en un medio para alcanzar metas.

Las creencias de autoeficacia son el resultado de la interacción de cuatro fuentes principales: experiencias anteriores, experiencias vicarias, persuasión verbal y estados fisiológicos (Bandura, 1986, citado en Ruiz, 2005).

Otros autores han estudiado la autoeficacia como un elemento global, inespecífico y estable; otros, como un elemento específico, vinculado a tareas y situaciones. No obstante, Bandura (1977, citado en Ruiz, 2005) aconseja que para evaluar la autoeficacia, esta se debe descomponer en las diferentes tareas o conductas que se requiere para el desempeño o desarrollo eficaz de una competencia.

La autoeficacia percibida de una persona dada se estimaría a partir de dos 2 indicadores: nivel de autoeficacia o número de conductas específicas que la persona es capaz de realizar; e intensidad o grado estimado en que la persona tiene autoconfianza para desempeñar cada una 
de las conductas o tareas señaladas de forma eficaz (Ruiz, 2005).

La autoeficacia se presenta, por tanto, como una variable interesante a introducir en el estudio de la discapacidad, puesto que se refiere a las creencias en las propias capacidades. Por ello, es lógico pensar que aspectos como las barreras arquitectónicas y la estigmatización que sufren las personas con limitaciones funcionales, pueden ser elementos especialmente relevantes en sus vidas para la consecución de las metas.

Por otro lado, la calidad de vida puede resultar un concepto más difícil de definir, pues a lo largo de la historia se han dado múltiples definiciones centradas en aspectos muy diferentes. En el campo de la psicología, la calidad de vida se centra principalmente en la percepción de bienestar de la persona, percepción que en nuestra sociedad se ve normalmente influida por elementos como la salud, el dinero, el apoyo social, etc. Se puede entender, por tanto, como un fenómeno subjetivo.

Las limitaciones funcionales físicas afectan a todas las áreas de la calidad de vida de las personas, pues son de carácter permanente, irreversibles y pueden conllevar a varias dificultades de adaptación. En este sentido, la calidad de vida va a estar determinada por el ajuste a las limitaciones cotidianas interpuestas, aunque también por síntomas somáticos como el dolor crónico o la percepción de control (Joyce-Moniz y Barros, 2007). Del mismo modo, las personas con limitaciones funcionales de origen físico suelen ver restringida su participación en las actividades de la vida diaria, producidas por las dificultades que se presentan en el entorno, ocasionando marginación y exclusión en muchos ámbitos.

De esta manera, la calidad de vida va a estar muy relacionada con las oportunidades que se ofrezcan, así como con su inclusión en la sociedad y el reconocimiento de sus derechos. Es aquí donde entra en juego la labor de la psicología en general y de la psicología social específicamente. Por ello, la calidad de vida ha sido elegida como otra de las variables a tener en cuenta en este estudio, pues parece fundamental el hecho de que una persona cuente con productos de apoyo y/o con recursos humanos como la prestación de asistencia personal.

Otra de las variables psicológicas objeto de este estudio son los estilos de afrontamiento y el estrés, teniendo como marco de referencia el proceso de ajuste que enfrentan las personas ante eventos que pueden dar como consecuencia una discapacidad, haciendo énfasis en la discapacidad física (Botero, 2013).

Quedando definido el afrontamiento como "aquellos esfuerzos cognitivos y conductuales constantemente cambiantes que se desarrollan para manejar las demandas específicas externas y/o internas, que son evaluadas como excedentes o desbordantes de los recursos del individuo" (Martín, Jiménez y Fernández-Abascal, 2001, p. 86). Las estrategias de afrontamiento como respuesta al estrés tienen por tanto dos funciones principales: la resolución del problema y el control emocional (Lazarus y Folkman, 1986).

Desde esta perspectiva, las personas con diversidad funcional, como seres humanos que se enfrentan a situaciones estresantes específicas debido a las limitaciones que la sociedad les interpone, tendrán necesidades de ajuste y requerirán el uso de estrategias de afrontamiento. Es lógico pensar, por tanto, que este estrés y estas estrategias de afrontamiento podrían cambiar si se les proporcionaran los recursos adecuados, como la prestación de asistencia personal, tema en el que se centra este estudio.

Así, el objetivo de este trabajo fue conocer la autoeficacia, la calidad de vida y los estilos de afrontamiento en personas con diversidad funcional de origen físico, comprobando la influencia de estas con el apoyo de asistencia personal y sin él.

La hipótesis que se planteó se basa en que las personas con diversidad funcional de origen físico que tienen la prestación de asistencia personal tienen mayor calidad de vida, más 
BÚSQUEDA - Enero / Junio de 2015 - No. 14 (7 - 18)

autoeficacia y mejor estilo de afrontamiento ante las diferentes situaciones cotidianas.

\section{Metodología}

El estudio utiliza un enfoque cuantitativo, transversal y el nivel de investigación es explicativo porque se encarga de buscar el por qué de los hechos mediante el establecimiento de relaciones causa-efecto.

Este trabajo ha centrado su metodología en las variables dependientes anteriormente citadas y explicadas; autoeficacia, estilos de afrontamiento y calidad de vida, relacionándolas con la variable independiente de prestación de asistencia personal.

Para el estudio y posterior análisis, se ha utilizado un cuestionario en formato online, realizado mediante el programa Google Drive. Este se ha compuesto de tres pruebas psicométricas diferentes para cada una de las tres variables: la Escala de Autoeficacia General (Baessler y Schwarzer, 1996) para el estudio de la autoeficacia, el cuestionario Whogol-Bref (Organización Mundial de la Salud, 1996) para el estudio de la calidad de vida y el cuestionario Brief-COPE (Carver, 1997, citado en Morá, Landero y González, 2009) para el estudio de los estilos de afrontamiento.

El acceso a los participantes fue posible gracias al Foro de Vida Independiente y Divertad, un grupo de personas que defienden el Movimiento de Vida Independiente en España, y que ha proporcionado el contacto con personas con diversidad funcional, con y sin asistencia personal.

De esta forma, una vez creado el cuestionario, de carácter anónimo y voluntario, se publicó el enlace en el Foro que crearon las personas que siguen el Movimiento de Vida Independiente y principales defensores de la prestación de asistencia personal, el Foro de Vida Independiente y Divertad; con el objetivo de recoger el mayor número de participantes que gozan de asistencia personal, de forma que los miembros del Foro que quisieron lo completaron, habiendo algunos que también lo mandaron a amigos o conocidos.

El único requisito que debían cumplir los participantes era tener el grado de discapacidad acreditado. Se obtuvieron 69 respuestas al cuestionario, de las cuales 65 acabaron siendo válidas y 4 tuvieron que ser eliminadas por no haber contestado todas las preguntas.

Una vez finalizada la recogida de datos, se realizó un análisis univariado para la descripción de la muestra y de las variables principales del estudio.

Posteriormente, se realizó un análisis bivariado para establecer las posibles diferencias entre los grupos de análisis: autoeficacia, calidad de vida y estilos de afrontamiento, y la variable prestación de asistencia personal. Para ello, se recurrió a una prueba de contraste de medias basada en el test no paramétrico $U$ de Mann-Whitney, para el contraste según existiera o no, la prestación de asistencia personal. El objetivo era observar si estas variables se veían afectadas por el hecho de que los participantes contaran o no con la misma.

En todos los casos se exigió una significación estadística del $5 \%(p<, 05)$.

Los análisis estadísticos se realizaron mediante el programa estadístico IBM SPSS Statistics, en su versión 22.0, con el apoyo de ciertos complementos de cálculo proporcionados por el programa Microsoft Excel.

\section{Resultados}

El estudio consta de 65 participantes pertenecientes a la población de personas con diversidad funcional de origen físico, con un mínimo de edad de 19 y un máximo de 65 años, y una media de edad de 44.22 años (desviación típica: 12.22). Dicha muestra está constituida por 27 hombres (41.5\%) y 38 mujeres (58.5\%). Con respecto al grado de dependencia, 11 participantes tienen un grado de dependencia bajo, (17.2\%), 13 (20.3\%) grado medio, y 40 
$(62.5 \%)$ grado alto, contando 25 participantes (38.5\%) con prestación de asistencia personal, mientras que los 40 restantes (61.5\%) no dispone de ella.

Respecto a la prestación de asistencia personal, la media de edad de los participantes que sí cuentan con dicha prestación, es de 42.88, mientras que la de los que no disponen de la misma es de 45.03 años.

Si se tiene en cuenta el sexo, el $48 \%$ de los participantes que sí cuentan con asistencia son hombres, en cambio, en el grupo de los que no cuentan con asistencia los hombres solo representan el $37.5 \%$.

En cuanto al grado de dependencia, el $84 \%$ de los que sí cuentan con asistencia personal tiene un grado de dependencia alta y el 16\%, media; no habiendo ningún sujeto con grado de dependencia baja. En el grupo de los participantes que no cuentan con la prestación, el $27.5 \%$ presenta grado de dependencia baja, el $22.5 \%$ media y el $47.5 \%$ alta.

En referencia a la variable de autoeficacia de los participantes, se ha analizado en función de que reciban o no prestación de asistencia personal. Los resultados muestran que los participantes que sí recibían la prestación tenían una media en autoeficacia de 33.80 (desviación típica 4.93) y los que no, de 30.23 (desviación típica 6.833); siendo esta diferencia estadísticamente significativa $[U(64)=344.50$, $\mathrm{p}=.035]$.

Para el estudio de la calidad de vida se han tenido en cuenta diferentes dominios: percepción en calidad de vida, satisfacción con la calidad de vida, salud física, salud psicológica, relaciones sociales y ambiente.

En cuanto a la percepción en calidad de vida, se ha contrastado que los participantes que sí cuentan con prestación de asistencia personal presentan una media en esta variable de 3.60 (desviación típica .764); resultando algo mayor que la de los participantes que no disfrutan de la prestación, teniendo estos una media en percepción de calidad de vida de 3.40 (desviación típica .900), aunque esta diferencia no es estadísticamente significativa [U (64) $=434.50, p=.346]$.

Por otro lado, se ha realizado el análisis de la satisfacción con respecto a la salud. Los resultados muestran que en la variable satisfacción con la salud los participantes con prestación tienen una media de 3.22 (desviación típica .795) y los que no cuentan con prestación, de 2.88 (desviación típica 1.017), siendo esta diferencia estadísticamente no significativa [U $(63)=367.50, p=.164]$.

En cuanto a la salud física, se ha contrastado que los participantes que sí gozan prestación de asistencia personal tienen una media en salud física de 15.04 (desviación típica 2.28); mientras que los que no cuentan con la prestación tienen una media de 12.78 (desviación típica 3.55). Esta diferencia sí es estadísticamente significativa [U (65) $=296.00, p=.006]$.

Al analizar la salud psicológica, los resultados muestran que aquellos participantes que cuentan con prestación tienen una media mayor que no la poseen, pues la media de los primeros es de 16.69 (desviación típica 1.93) frente a la media de 14.48 (desviación típica 2.89) de los segundos, siendo en este caso la diferencia estadísticamente significativa [U (65) $=276.00$, $\mathrm{p}=.002]$.

En cuanto a las relaciones sociales, se ha contrastado que los participantes que se beneficiaban de la prestación de asistencia personal tienen una media de 14.77 (desviación típica 3.37), mientras que los que no la tenían presentan una media de 12.60 (desviación típica 3.87). Esta diferencia es estadísticamente significativa [ $U(65)=338.00, p=.028]$.

El último dominio de la calidad de vida es el ambiente. Se ha contrastado que los participantes que cuentan con prestación tienen una media de 14.40 en el ambiente (desviación típica 2.31) frente a una media de 12.98 (desviación típica 
BÚSQUEDA - Enero / Junio de 2015 - No. 14 (7 - 18)

3.06) en el caso de los participantes que no disfrutan de la prestación, aunque esta diferencia resulta estadísticamente no significativa [U $(65)=358.00, p=.055]$.

Para el estudio de los estilos de afrontamiento, se ha usado el cuestionario Brief-COPE que se compone de 13 subescalas, que a su vez se agrupan en cuatro escalas globales, que son las que se han tenido en cuenta para el análisis bivariado: afrontamiento cognitivo, afrontamiento de apoyo social, bloqueo del afrontamiento y afrontamiento espiritual.

Al analizar el primer dominio, el afrontamiento cognitivo, se ha contrastado que los participantes que se beneficiaban prestación de asistencia personal presentan una de media de 2.44 (desviación típica .624), resultando menor que la de los participantes que no la poseían teniendo estos una media de 2.60 (desviación típica .766), aunque esta diferencia no es estadísticamente significativa [U (65) $=403.50, p=.191]$.

En cuanto al afrontamiento de apoyo social, se observa que los participantes cobijadas con la prestación de asistencia personal tienen una media de 1.38 (desviación típica .678), mientras que los que no, presentan una media de 1.33 (desviación típica .619). Esta diferencia no es estadísticamente significativa [U (65) $=471.500$, $\mathrm{p}=.700]$.

Al analizar el dominio bloqueo del afrontamiento, los resultados muestran que aquellos participantes que cuentan con prestación tienen una media de .94 (desviación típica .342) frente a la media de 1.04 (desviación típica .484) de los excluidos de la prestación, resultando en este caso la diferencia estadísticamente no significativa [U (65) $=438.500, p=.403]$.

Por último, al realizar el análisis del dominio afrontamiento espiritual, se contrasta que los participantes que sí contaban con prestación de asistencia personal presentan una media de .66 (desviación típica .850); resultando menor que la de las personas que no contaban con la prestación, teniendo estos una media de 1.00 (desviación típica 1.176), aunque esta diferencia no es estadísticamente significativa [U (65) $=442.00, p=.406]$.

En cuanto a las diferencias según el estilo de afrontamiento, se observa que las puntuaciones más altas se dan en afrontamiento cognitivo, seguido de afrontamiento por apoyo social, bloqueo de afrontamiento y, por último, afrontamiento espiritual.

Respecto al estudio de la fiabilidad, esta muestra que la autoeficacia tiene un alfa de Cronbach de .917, siendo por tanto la escala bastante fiable. Los dominios del cuestionario Whoqol-Bref ofrecen unos valores más bajos, resultando el alfa de Cronbach de .832 en el dominio salud física, .841 en salud psicológica, .698 en relaciones sociales y .847 en ambiente. Sin embargo, la escala global de calidad de vida presenta la fiabilidad más alta de todas las pruebas del estudio, con un alfa de Cronbach de .927. Se puede decir, por tanto, que el cuestionario de calidad de vida también resulta fiable.

Tabla 1. Estilos de afrontamiento de los participantes de la muestra según el Brief-COPE

Estadísticos descriptivos

\begin{tabular}{lcccc}
\hline & N Media & Desviación típica & Mínimo & Máximo \\
\hline EAF.Afront.Cog & 652.5436 & .71460 & .83 & 3.83 \\
EAF.Afront.Apoyo.So & 651.3538 & .63772 & .00 & 2.83 \\
EAF.Bloq.Afront & 651.0077 & .43544 & .17 & 2.33 \\
EAF.Afront.Espi & 65.8692 & 1.06901 & .00 & 3.00 \\
\hline
\end{tabular}

Fuente: Datos obtenidos del análisis en SPSS 
Por último, las escalas del cuestionario de estilos de afrontamiento son las que presentan unos valores de alfa de Cronbach más bajos, siendo .653 en la escala de afrontamiento cognitivo, .771 en la escala de afrontamiento de apoyo social, .451 en la escala de bloqueo de afrontamiento y .824 en la escala de afrontamiento espiritual. De este modo, resulta la prueba con menor fiabilidad, a causa de los bajos valores de las escalas de bloqueo de afrontamiento y de afrontamiento cognitivo.

\section{Discusión}

Las variables que se han elegido para este estudio son la autoeficacia, los estilos de afrontamiento y la calidad de vida; y en el marco de la Teoría Social Cognitiva, la autoeficacia se refiere a:

La evaluación que efectúa una persona de su capacidad y competencia para llevar a cabo una tarea, alcanzar una meta o vencer un obstáculo [...] La valoración de la capacidad se hace no solo a nivel psicosocial, sino además a nivel físico (enfermedad, dolor...). (Ruiz, 2005, p. 20).

Por ello, resultaba interesante introducir la autoeficacia como variable en el estudio para poder comprobar si esta percepción de capacidad se veía influida o no por el apoyo social en las personas con diversidad funcional de origen físico.

Tal y como se expone en el apartado anterior, el cuestionario de autoeficacia utilizado resultó tener una fiabilidad alta (alfa de Cronbach .917), por lo que se puede decir que en este caso la variable cumple la hipótesis de partida: las personas del estudio que cuentan con asistencia personal tienen un mayor nivel de autoeficacia que las que no. Esto concuerda con todas las teorías que afirman que el aspecto social influye directamente en la valoración que hacemos de nosotros mismos, tanto a nivel de autoestima y autoconcepto como a nivel de autoeficacia.

Asimismo, se pueden relacionar los resultados con la tesis realizada por Ruiz (2005), la cual analiza fundamentalmente el papel que juega la comparación social en el afrontamiento de desempleo en las personas con diversidad funcional de origen físico. De ella se obtuvo como resultado la importancia de la autoeficacia percibida en las estrategias de mejora de la identidad social y de la intención de realizar acciones (individuales o colectivas) para evitar el desempleo laboral.

Esto quiere decir que en las personas con diversidad funcional de origen físico la autoeficacia se revela como un elemento clave para sentirse incluidas en la sociedad y para realizar acciones y alcanzar metas, lo que reafirma la relevancia de haber elegido la autoeficacia como una de las variables a estudiar en este trabajo y la estrecha relación entre la discapacidad, lo social y la autoeficacia.

Otra de las variables de este estudio era la calidad de vida. Resultaba fundamental incluirla, dado que el objetivo era observar la influencia que la prestación de asistencia personal podía tener en las personas con diversidad funcional de origen físico.

Para el estudio de la calidad de vida, se han tenido en cuenta diferentes dominios: percepción en calidad de vida, satisfacción con la calidad de vida, salud física, salud psicológica, relaciones sociales y ambiente. Las diferencias entre las personas que tenían prestación de asistencia personal y las que no, no resultan estadísticamente significativas en el caso de los dominios percepción en calidad de vida, satisfacción con la calidad de vida y ambiente, por tanto, no podemos decir que el hecho de contar o no con la prestación suponga una diferencia en estas variables.

Sin embargo, sí se encuentran diferencias estadísticamente significativas en los dominios de salud física (media de 15.04 en los participantes que cuentan con prestación y de 12.78 en los que no); en salud psicológica (media de 16.69 en los participantes que sí, frente a la media de 14.48 en los participantes que no); y en relaciones sociales (14.77 en el caso de los participantes 
BÚSQUEDA - Enero / Junio de 2015 - No. 14 (7 - 18)

que contaban con asistencia personal y 12.60 en los que no), puesto que la fiabilidad de todos los dominios es aceptable (el alfa de Cronbach es de .698 en el dominio relaciones sociales) y la fiabilidad global del test resulta en un alfa de Cronbach de .927. Se puede decir que en el caso de las variables salud física, salud psicológica y relaciones sociales se cumple la hipótesis inicial.

Es decir, los participantes que contaban con la prestación de asistencia personal perciben niveles más altos de salud física, salud psicológica y relaciones sociales, aunque no se observan diferencias significativas en el resto de dominios (percepción en calidad de vida, satisfacción con la calidad de vida y ambiente).

Esto podría deberse a que el hecho de contar o no con prestaciones que mejoren la vida de las personas con discapacidad no es la única variable determinante en su calidad de vida. Una variable que podría ser muy influyente es el grado de dependencia de estas personas, pues no es lo mismo la percepción de calidad de vida de una persona con dificultades leves para andar que la de una persona con movilidad totalmente reducida.

También, podría ser una variable relevante el estudio del nivel socioeconómico, pues es un factor muy determinante en la vida de las personas con discapacidad. Sería, por tanto, interesante realizar un nuevo estudio que incluyera estos factores, para contrastar si los resultados se deben a la influencia de estas variables que en este estudio no han podido ser tenidas en cuenta.

La última variable estudiada en este trabajo han sido los estilos de afrontamiento, que fue elegida por ser una de las variables determinantes en los sucesos estresantes de todo tipo, como pueden ser las limitaciones y barreras con las que se encuentran las personas con discapacidad, constantemente en su vida diaria. La hipótesis de partida era, por tanto, que las personas que cuentan con ayudas de asistencia personal (que es una forma de prestación social) tendrían mejores estilos de afrontamiento.

Las cuatro escalas globales que se han analizado han sido: afrontamiento cognitivo, afrontamiento de apoyo social, bloqueo del afrontamiento y afrontamiento espiritual. Los resultados muestran que las diferencias entre las personas que contaban con la prestación de asistencia personal en las puntuaciones de cada dominio son pequeñas y ninguna es estadísticamente significativa.

Además, en el estudio de la fiabilidad se observa que ningún dominio tiene valores de alfa de Cronbach muy altos (.653 en afrontamiento cognitivo, .771 en afrontamiento de apoyo social, .451 en bloqueo de afrontamiento y .824 en afrontamiento espiritual), por lo que al menos dos de ellos no parecen fiables.

Sin embargo, sí se observan diferencias entre los diferentes estilos de afrontamiento: el afrontamiento cognitivo prevalece en primer lugar, seguido del afrontamiento por apoyo social y del bloqueo de afrontamiento, quedando el afrontamiento espiritual en último lugar.

\section{Conclusiones}

Se pretendía observar si este apoyo, la prestación de asistencia personal, es relevante para la vida de las personas participantes de este estudio. Otra de las causas ha sido el hecho de que no es una prestación muy conocida ni solicitada por lo que no existen muchos estudios científicos que hayan trabajado con esta variable a la hora de estudiar este apoyo social, por lo que este trabajo podría aportar una perspectiva nueva desde la psicología.

De tal forma, que la hipótesis inicial acerca de la variable estilos de afrontamiento no se ve cumplida, pues no se observan diferencias en la elección de los estilos de afrontamiento entre las personas que contaban con asistencia personal y las que no.

Dado que la elección de los estilos de afrontamiento no depende de la prestación de asistencia personal sino de otras variables, y que los participantes prefieren el afrontamiento cognitivo o el afrontamiento por apoyo social que el bloqueo 
de afrontamiento o el afrontamiento espiritual , sería necesario ampliar el estudio a una muestra más grande y más variada para observar si la causa de que no se den diferencias es que el grupo elegido tiene variabilidad intragrupal. Podría deberse a que los participantes pertenecen a un colectivo determinado, pues se trata de participantes que colaboran con el Movimiento de Vida Independiente o son cercanos a ello.

Por otro lado, en el caso de la variable calidad de vida, los participantes que contaban con la prestación de asistencia personal presentaron niveles más altos de percepción de salud física, salud psicológica y relaciones sociales, pero no se observan diferencias significativas en los dominios percepción en calidad de vida, satisfacción con la calidad de vida y ambiente.

La hipótesis inicial se cumpliría solo a medias, no pudiendo afirmar por tanto que la calidad de vida como variable global es mayor en los participantes que contaban con la prestación, pero sí en los dominios salud física, salud psicológica y relaciones sociales.

El estudio realizado confirma que las personas del estudio que contaban con asistencia personal tienen un mayor nivel de autoeficacia que las que no, cumpliendo así la hipótesis inicial en cuanto a la variable autoeficacia.

Como se ha comentado, podría ser interesante realizar otro estudio que incluyera variables como el grado de dependencia o el nivel socioeconómico, para comprobar si los resultados se deben a la influencia de estas variables, que en este estudio no han podido ser tenidas en cuenta.

\section{Referencias bibliográficas}

Baessler, J. y Schwarcer, R. (1996). Evaluación de la autoeficacia: Adaptación española de la escala de Autoeficacia General. Ansiedad y Estrés, 2: 1-8.
Bandura, A. (1999). Social cognitive theory: An agentic perspective. Asian Journal of Social Psychology, 2: 21-41.

Boletín Oficial del Estado (2006). Ley 39/2006, de 14 de diciembre, de Promoción de la Autonomía Personal y Atención a las personas en situación de dependencia. Madrid, España.

Boletín Oficial del Estado (2013). Texto refundido de la Ley General de Derechos de las personas con Discapacidad y su inclusión social. Madrid, España: Autor.

Botero, P. A. (2013) Discapacidad y estilos de afrontamiento: una revisión teórica. Revista Vanguardia Psicológica, 3(2): 196-214.

Canto, J. E. (1998). Autoeficacia y educación. Educación y Ciencia, 2(4): 45-53.

Instituto de Mayores y Servicios Sociales (2015). Personas beneficiarias y agrupación prestaciones. Madrid: Autor. Recuperado de: http://dependencia.imserso.es/ InterPresent1/groups/imserso/documents/ binario/im_062034.pdf

Joyce-Moniz, L., y Barros, L. (2007). Psicología de la enfermedad para cuidados de la salud. Desarrollo e intervención. México: Manual Moderno.

Lazarus, R. S. y Folkman, S. (1986). Estrés y procesos cognitivos. Barcelona: Martínez Roca.

Martín, M. D.; Jiménez, M. P. y FernándezAbascal, E. (2000). Estudio sobre la Escala de Estilos y Estrategias de Afrontamiento. Revista Española de Motivación y Emoción, 3(4): 85-98.

Morán, C., Landero, R., y González, M.T. (2009). COPE-28: un análisis psicométrico de la versión en español del Brief COPE. Universitas Psychologica, 9(2): 543-552. 
BÚSQUEDA - Enero / Junio de 2015 - No. 14 (7 - 18)

Organización de las Naciones Unidas (2006). Convención de los derechos humanos de las personas con discapacidad. Nueva York.

Palacios, A. y Romañach, J. (2007). El modelo de la diversidad. La Bioética y los Derechos Humanos como herramientas para alcanzar la plena dignidad en la diversidad funcional. Ediciones Diversitas-AIES. Recuperado de: https://www.fuhem.es/media/ecosocial/ file/Boletin\%20ECOS/ECOS\%20CDV/ Boletin_8/ModeloDversidad.pdf

Ruiz, J. (2005). La Discapacidad como estigma: un análisis psicosocial del afrontamiento del desempleo de las personas con discapacidad física. Revista REDSI Red Social Interactiva, (6). Recuperado de: http://sid.usal.es/idocs/F8/ART6925/ desempleofisicos.pdf
Soto, N. y Vasco, C.E. (2008). Representaciones sociales y discapacidad. Revista académica Hologramática, 8(1): 3-22.

Verdugo, M.A. (s.f.). La concepción de la discapacidad en los modelos sociales. Recuperado de: http://www.um.es/discatif/ TEORIA/Nerdugo-ModelosSoc.pdf

Verdugo, M.A., Vicent, C., Campo, M. y Jordan de Urríes, B. (2001). Definiciones de Discapacidad en España: un análisis de la normativa y la legislación más relevante. Salamanca, España: Servicio de Información sobre Discapacidad. Recuperado de: http://sid.usal.es/idocs/ F8/8.4.1-5021/8.4.1-5021.PDF

World Health Organization (1996). WHOQOLBREF Introduction, administration, scoring and version of the assessment. Ginebra: Author. Recovered from: http://www.who. int/mental_health/media/en/76.pdf 